\title{
Decreased expression of prenyl diphosphate synthase subunit 2 correlates with reduced survival of patients with gastric cancer
}

\author{
Mitsuro Kanda ${ }^{\text {* }}$, Shuji Nomoto ${ }^{1}$, Hisaharu Oya ${ }^{1}$, Ryoji Hashimoto ${ }^{1}$, Hideki Takami ${ }^{1}$, Dai Shimizu${ }^{1}$, \\ Fuminori Sonohara', Daisuke Kobayashi', Chie Tanaka', Suguru Yamada', Tsutomu Fujii', Goro Nakayama', \\ Hiroyuki Sugimoto', Masahiko Koike', Kenta Murotani ${ }^{2}$, Michitaka Fujiwara' ${ }^{1}$ and Yasuhiro Kodera ${ }^{1}$
}

\begin{abstract}
Background: Identification of novel molecular biomarkers will improve the management of patients with gastric cancer (GC). Prenyl diphosphate synthase subunit 2 (PDSS2) is required for coenzyme Q10 biosynthesis and acts as a tumor suppressor; however, the role and regulatory mechanisms of PDSS2 in GC are not understood. The aim of this study was to determine expression status and regulatory mechanisms of PDSS2 in GC.

Methods: Associations between expression and methylation of PDSS2 were evaluated using GC cell lines. The clinical significance of PDSS2 expression was evaluated using 238 pairs of surgically resected gastric tissues with subgroup analysis based on GC subtypes.

Results: The expression of PDSS2 mRNA was decreased in 73\% of GC cell lines compared with the control non-cancerous cell. The PDSS2 promoter was hypermethylated in cells with decreased PDSS2 expression, and treating these cells with a methylation inhibitor reactivated PDSS2 expression. GC tissues expressed significantly lower mean levels of PDSS2 mRNA compared with adjacent normal tissues $(P<0.001)$. The expression pattern of PDSS2 protein was consistent with that of its mRNA. The decrease of PDSS2 mRNA expression in GC tissues (less than half the level of expression detected in the corresponding normal adjacent tissues) correlated significantly with elevated levels of carbohydrate antigen $19-9(P=0.015)$, lymph node metastasis $(P=0.022)$, and shorter recurrence-free survival after curative resection $(P=0.022)$. Further, multivariate analysis identified PDSS2 mRNA expression as an independent prognostic factor (hazard ratio 1.95, 95\% confidence interval 1.22-3.09, $P=0.005$ ), and its expression pattern and prognostic significance were similar among three GC subtypes.

Conclusions: PDSS2 encodes a putative tumor suppressor, and we show here that its expression was regulated by hypermethylation of its promoter in GC cells. Inhibition of PDSS2 mRNA expression may serve as a novel biomarker of all types of GC.
\end{abstract}

Keywords: Gastric cancer, Prenyl diphosphate synthase subunit 2, Expression, methylation, Subtype

\section{Background}

Although the incidence of gastric cancer $(\mathrm{GC})$ is declining in most developed countries, it remains one of the most common causes of cancer-related death worldwide [1-3]. Appropriate stratification of patients is a pivotal aspect of individualized treatment, leading to reducing mortality from this cancer $[4,5]$.

\footnotetext{
* Correspondence: m-kanda@med.nagoya-u.ac.jp

1 Department of Gastroenterological Surgery (Surgery II), Nagoya University Graduate School of Medicine, 65 Tsurumai-cho, Showa-ku, Nagoya 466-8550, Japan
} Full list of author information is available at the end of the article
According to its epidemiology, pathology, and location in the body, GC is recognized as three distinct malignancies arising in the same organ [6-8]. Shah et al. [9] proposed a convincing classification of GC according to histopathologic and anatomic criteria as follows: (1) proximal nondiffuse GC where the tumor is located mainly in the gastric cardia with evidence of precursor glandular dysplasia or in situ carcinoma in the presence of chronic inflammation, usually without atrophy; (2) diffuse GC, which may be located anywhere in the stomach with no apparent 
gastritis that exhibits an entirely diffuse pattern of infiltration of cells with a poorly differentiated phenotype; and (3) distal nondiffuse GC, which is located mainly in the distal stomach with evidence of chronic gastritis that is predominantly differentiated or exhibits an intestinal phenotype. In this study, they demonstrated that the three GC subtypes are distinguished by their gene expression profiles. Therefore, the genetic diversity of GC subtypes should be considered in studies of genetic and epigenetic alterations related to gastric carcinogenesis and progression.

Prenyl diphosphate synthase subunit 2 (PDSS2) was identified in 2005 [10], and evidence indicates that it acts as a tumor suppressor $[11,12]$. PDSS2 is required for the synthesis of coenzyme Q10 (CoQ10) [13,14], which is synthesized in the mitochondrial inner membrane and plays a vital role in the mitochondrial respiratory chain, pyrimidine nucleoside biosynthesis, and modulation of apoptosis [15]. PDSS2 resides within the chromosomal locus 6q16.3-21, a site of frequent microsatellite DNA instability and loss of heterozygosity (LOH) in GC $[16,17]$, supporting its role as a tumor suppressor in gastric epithelial cells. Moreover, PDSS2 may suppress the development of malignant melanomas and lung cancers $[11,12]$. Moreover, Chen et al. reported that enforced overexpression of PDSS2 leads to apoptosis in a GC cell line by causing cell cycle arrest in the G0/G1 phase [18]. These reports led us to make a hypothesis that PDSS2 is a potential GC-related gene and a candidate of novel clinically-relevant prognostic marker of GC.

In this study, expression and methylation status of PDSS2 in $\mathrm{GC}$ were determined to evaluate the clinical significance and regulatory mechanisms of PDSS2 expression in GC. Our results indicate that $P D S S 2$ expression provides a potential clinical biomarker of the progression and recurrence of GC.

\section{Material and methods Ethics}

This study conformed to the ethical guidelines of the World Medical Association Declaration of Helsinki-Ethical Principles for Medical Research Involving Human Subjects and has been approved by the Institutional Review Board of Nagoya University, Japan. Written informed consent for usage of clinical samples and data, as required by the institutional review board, was obtained from all patients [4].

\section{Sample collection}

Eleven GC cell lines (H111, KATOIII, MKN1, MKN28, MKN45, MKN74, NUGC2, NUGC3, NUGC4, SC-2-NU and SC-6-LCK) and CCL-241 (non-cancerous cell line derived from the small intestine) were obtained from the American Type Culture Collection (Manassas, VA, USA) or Tohoku University, Japan. The GC cell lines were cultured at $37^{\circ} \mathrm{C}$ in RPMI-1640 (Sigma-Aldrich, St. Louis,
MO, USA) supplemented with $10 \%$ fetal bovine serum in an atmosphere containing $5 \% \mathrm{CO}_{2}$. For CCL-241, $30 \mathrm{ng} / \mathrm{ml}$ of the epidermal growth factor (Sigma-Aldrich) was added in the medium. Primary GC tissues and corresponding normal adjacent tissues were collected from 238 patients who underwent gastric resection for GC at Nagoya University Hospital between 2001 and 2012. Patients who received neoadjuvant therapy were excluded because it was difficult to obtain cancer cells from scarred tissues. Specimens were classified histologically using the 7th edition of the Union for International Cancer Control (UICC) classification [19]. Relevant clinicopathological parameters were acquired from medical records. To evaluate whether the expression level of PDSS2 correlated with tumor phenotype, patients were categorized into three groups according to the definition of GC subtypes according to the criteria of Shah et al. [9] as follows: proximal nondiffuse, diffuse, and distal nondiffuse type. Since 2006, adjuvant chemotherapy using S-1 (an oral fluorinated pyrimidine) is administered to all UICC stage II-III patients with GC unless contraindicated by the patient's condition [20]. Patients were followed at least once every 3 months for 2 years after surgery and then every 6 months for 5 years or until death. Physical examination, laboratory tests, and enhanced computed tomography (chest and abdominal cavity) were performed at each visit. Chemotherapy for patients with distant metastasis or after recurrence was determined by physician's discretion.

Tissue samples were immediately frozen in liquid nitrogen and stored at $-80^{\circ} \mathrm{C}$. Tumor samples without necrotic areas (approximately $5 \mathrm{~mm}^{2}$ ) were extracted by gross observation and only samples confirmed to comprise more than $80 \%$ tumor components by H\&E staining were included in this study. Corresponding normal adjacent gastric mucosa samples $>5 \mathrm{~cm}$ from the edge of the tumors were obtained from the same patient [21].

\section{Quantitative real-time reverse-transcription polymerase chain reaction (qRT-PCR)}

Total RNAs (10 $\mu \mathrm{g}$ per sample) were isolated from 11 GC cell lines, CCL-241, 238 primary GC tissues and corresponding normal adjacent tissues were used to generate CDNAs, which were amplified using specific PCR primers (Additional file 1: Table S1). Real-time detection of $\mathrm{SYBR}^{\circ}$ Green fluorescence intensity was conducted using an ABI StepOnePlus Real-Time PCR System (Applied Biosystems, Foster City, CA, USA). The expression of GAPDH mRNA was quantified in each sample for standardization. The qRT-PCR reactions in each sample were performed in triplicate. The expression level of each sample is presented as the value of the PDSS2 amplicon divided by that of GAPDH [22]. PDSS2 mRNA expression was defined as decreased in GC tissues 
when its level was less than half that of the corresponding normal adjacent tissue.

\section{Analysis of the promoter region of PDSS2}

The nucleotide sequence of the PDSS2 promoter region was analyzed to determine the presence or absence of CpG islands defined as follows: at least a 200-bp region of DNA with a high GC content $(>50 \%)$ and an Observed CpG/Expected CpG ratio $\geq 0.6$ [23]. We used CpG Island Searcher software (http://cpgislands.usc.edu/) to determine the locations of CpG islands [24].

\section{Methylation-specific PCR (MSP) and bisulfite sequence analysis}

PDSS2 possesses a CpG island near its promoter region, and we hypothesized that aberrant methylation is responsible for regulating the transcription of PDSS2 in GC. DNA samples from $11 \mathrm{GC}$ cell lines treated with bisulfite were subjected to MSP and nucleotide sequence analysis [25]. The primer sequences used for MSP and bisulfite sequencing are listed in Additional file 1: Table S1.

\section{5-Aza-2'-deoxycytidine (5-aza-dC) treatment}

To assess the relation of promoter hypermethylation to PDSS2 transcription, GC cells $\left(1.5 \times 10^{6}\right)$ were treated with 5-aza-dC (Sigma-Aldrich) to inhibit DNA methylation and cultured for 6 days with medium changes on days 1,3 , and 5. RNA was extracted, and RT-PCR was performed as described [7].

\section{Immunohistochemistry (IHC)}

IHC analysis of the localization of PDSS2 was performed using a mouse monoclonal antibody against PDSS2 (ab119768, Abcam, Cambridge, UK) diluted 1:150 in antibody diluent (Dako, Glostrup, Denmark) to probe 30 representative formalin-fixed and paraffin-embedded sections of well-preserved GC tissue described previously [3]. Staining patterns were compared between GCs and the corresponding normal adjacent tissues. To avoid subjectivity, the specimens were randomized and coded before analysis by two independent observers who were unaware of the status of the samples. Each observer evaluated all specimens at least twice to minimize intraobserver variation [7].

Evaluation of the clinical significance of PDSS2 expression Correlations between the pattern of PDSS2 mRNA expression and clinicopathological parameters were evaluated according to the differences among the three GC subtypes. Subgroup analysis of survival according to GC subtype was performed to determine the influence of PDSS2 expression on patients' outcomes.

\section{Statistical analysis}

Relative levels of mRNA expression (PDSS2/GAPDH) between $\mathrm{GC}$ and adjacent normal tissues were analyzed using the Mann-Whitney $U$ test. The $\chi^{2}$ test was used to analyze the significance of the association between the expression and methylation status of PDSS2 and clinicopathological parameters. Disease-specific and disease-free survival rates were calculated using the Kaplan-Meier method, and the difference in survival curves was analyzed using the log-rank test. We performed multivariate regression analysis to detect prognostic factors using the Cox proportional hazards model, and variables with $P<0.05$ were entered into the final model. All statistical analyses were performed using JMP 10 software (SAS Institute Inc, Cary, NC, USA). A value of $P<0.05$ was considered statistically significant.

\section{Results}

Identification of a CpG island in the PDSS2 promoter

A CPG island was identified in the PDSS2 promoter region using the $\mathrm{CpG}$ Island Searcher. The properties of the $\mathrm{CpG}$ island are as follows: $1655 \mathrm{bp}, 55.9 \% \mathrm{GC}$, and 0.70 Observed CpG/Expected CpG ratio (Figure 1A). Therefore, we hypothesized that hypermethylation of the CPG islands regulates the expression of PDSS2 in GC.

\section{PDSS2 mRNA expression and methylation status in GC cell lines}

Significant decreases in PDSS2 mRNA levels were detected in seven $(73 \%)$ of $11 \mathrm{GC}$ cell lines compared with the expression level of the CCL-241 cell (Data for GC tissues are described below). There was no apparent difference in expression levels between cell lines derived from differentiated and undifferentiated GCs (Figure 1B). Hypermethylation of the PDSS2 promoter was detected in MKN1, SC-2-NU, KATOIII, MKN45, and NUGC3 cells (Figure 1B). To determine whether hypermethylation of the PDSS2 promoter inhibited transcription, mRNA expression levels were compared before and after treating cells with the methylation inhibitor 5-aza-dC. PDSS2 mRNA levels were restored in cells with down-regulated PDSS2 expression accompanying hypermethylation after 5 -aza-dC treatment (Figure 1B), indicating that promoter hypermethylation inhibited PDSS2 transcription in GC. Representative chromatograms of the sequence analysis of the PDSS2 promoter region in MKN28 (complete methylation) and NUGC4 (absence of methylation) cells are shown in Figure $1 \mathrm{C}$.

\section{Patient characteristics}

The patient population included 179 males and 59 females aged from 20 to 84 years $(65.3 \pm 11.7$ years, mean \pm standard deviation). Pathologically, 139 and 99 patients were diagnosed with undifferentiated and differentiated GC, 


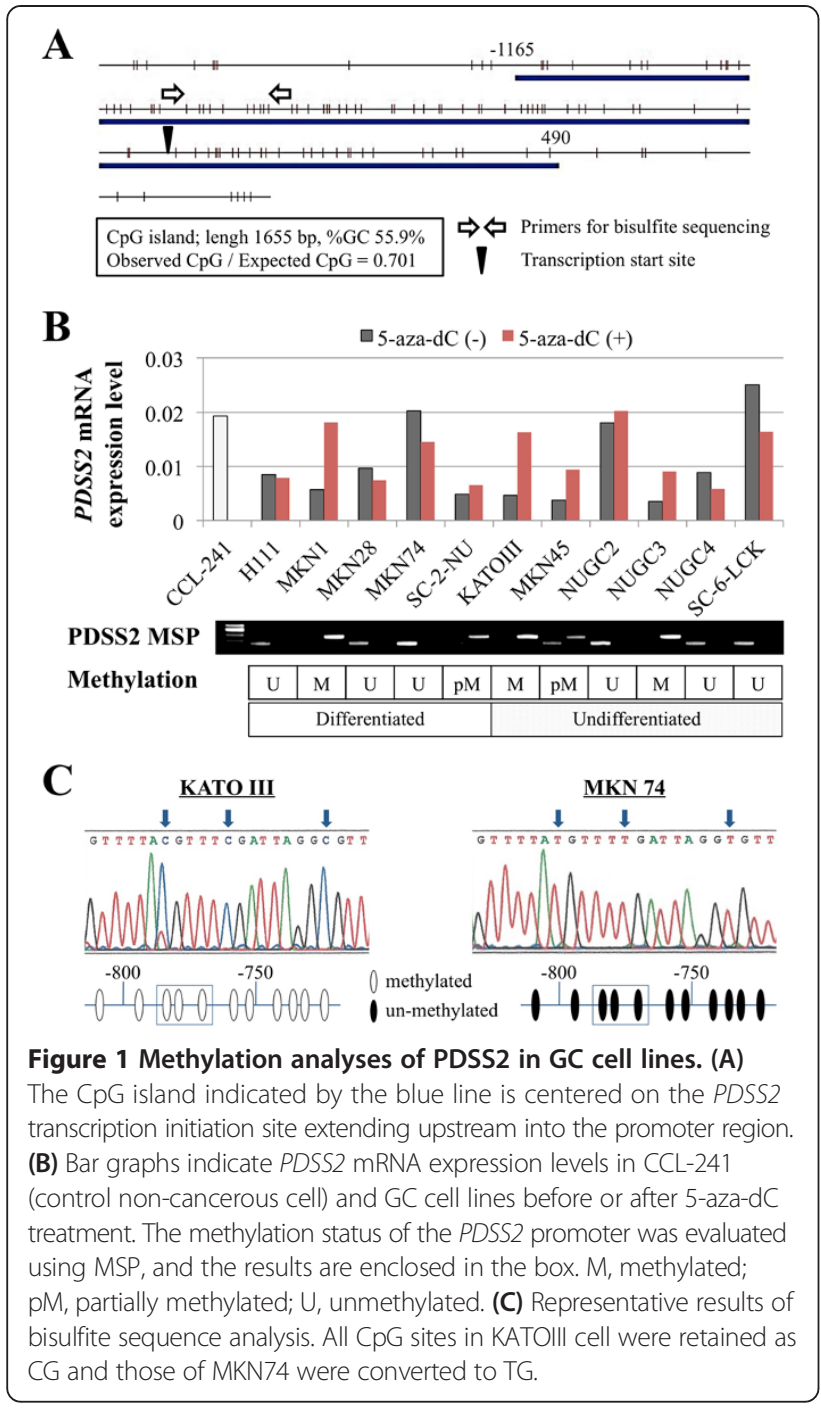

respectively. Patients were classified into the three GC phenotypes as follows: nondiffuse, 54; diffuse, 48; and distal nondiffuse, 136. According to the 7th edition of the UICC classification, 58, 40, 71 and 69 patients were in stages I, II, III and IV, respectively. One hundred sixtyfour patients in stages I-III underwent R0 resection. Sixty of the 69 patients in UICC stage IV were diagnosed as stage IV due to positive peritoneal lavage cytology, localized peritoneal metastasis or distant lymph node metastasis. Eight of patients in stage IV had synchronous liver metastasis, one had lung metastasis, and they underwent gastrectomy aimed to control bleeding or obstruction to the passage of food.

\section{Expression levels of PDSS2 mRNA and protein in surgically resected tissues}

The mean expression level of PDSS2 mRNA was lower in $\mathrm{GC}$ tissues compared with that of normal adjacent tissues $(P<0.001)$; however, there was no significant difference in PDSS2 mRNA expression levels between patients with undifferentiated or differentiated GC (Figure 2A). The expression pattern of PDSS2 was evaluated using IHC. Representative cases with reduced PDSS2 staining of GC tissues are shown in Figure 2B. Overall, the staining patterns of PDSS2 were consistent with the qRT-PCR data.

\section{Prognostic implications of PDSS2 mRNA expression levels} The expression of PDSS 2 mRNA in GC tissues was decreased in $76(32 \%)$ of 238 patients (less than half the level of expression detected in the corresponding normal adjacent tissues). The disease-specific survival rate of patients with decreased levels of PDSS2 mRNA in GCs was significantly lower compared with those without (5-year survival rates, $36 \%$ and $64 \%$, respectively, $P<0.001$, Figure 3A). Decreased levels of PDSS2 mRNA in GCs were significantly associated with carbohydrate antigen (CA) 19-9 > $37 \mathrm{IU} / \mathrm{ml}$ and lymph node metastasis (Table 1). Univariate analysis of disease-specific survival showed that GC subtype (proximal nondiffuse or diffuse), CA 19-9 > $37 \mathrm{IU} / \mathrm{ml}$, tumor size $(\geq 50 \mathrm{~mm})$, pT4, undifferentiated tumor, lymphatic involvement, vessel invasion, invasive growth, lymph node metastasis, positive peritoneal lavage cytology, and decreased PDSS2 mRNA expression in GC tissues were significant prognostic factors of adverse outcomes. Multivariate analysis identified decreased PDSS2 mRNA expression as an independent prognostic factor (hazard ratio 1.95, 95\% confidence interval 1.22-3.09, $P=0.005$, Table 2 ). The proportional hazards assumption

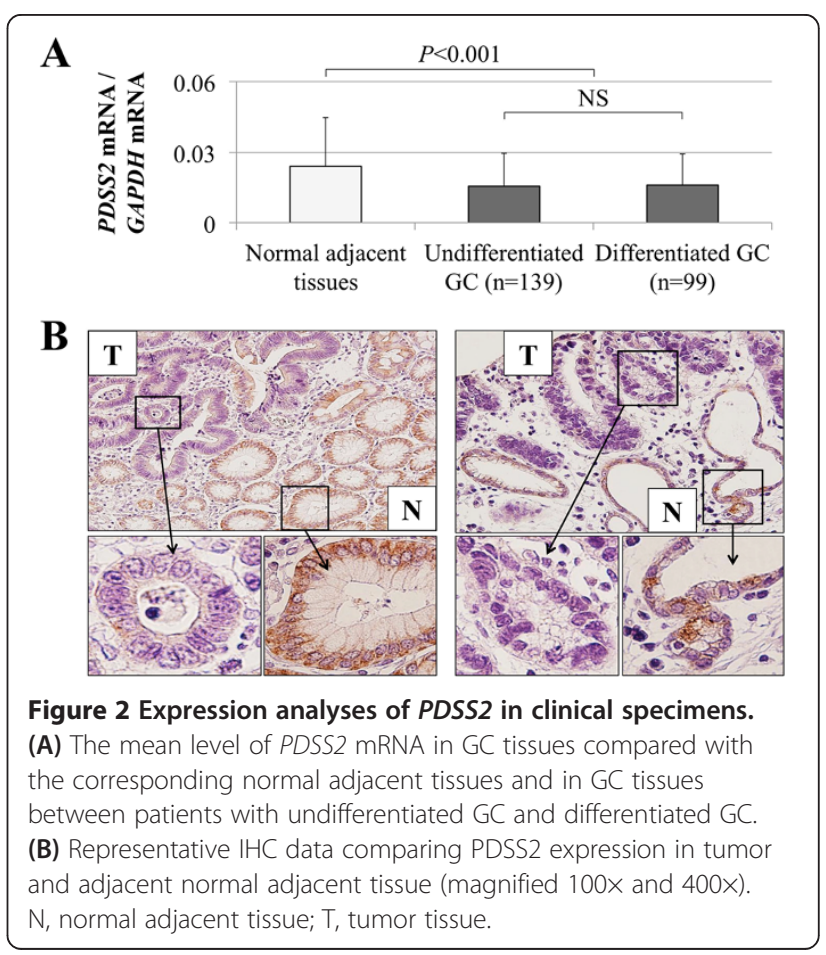




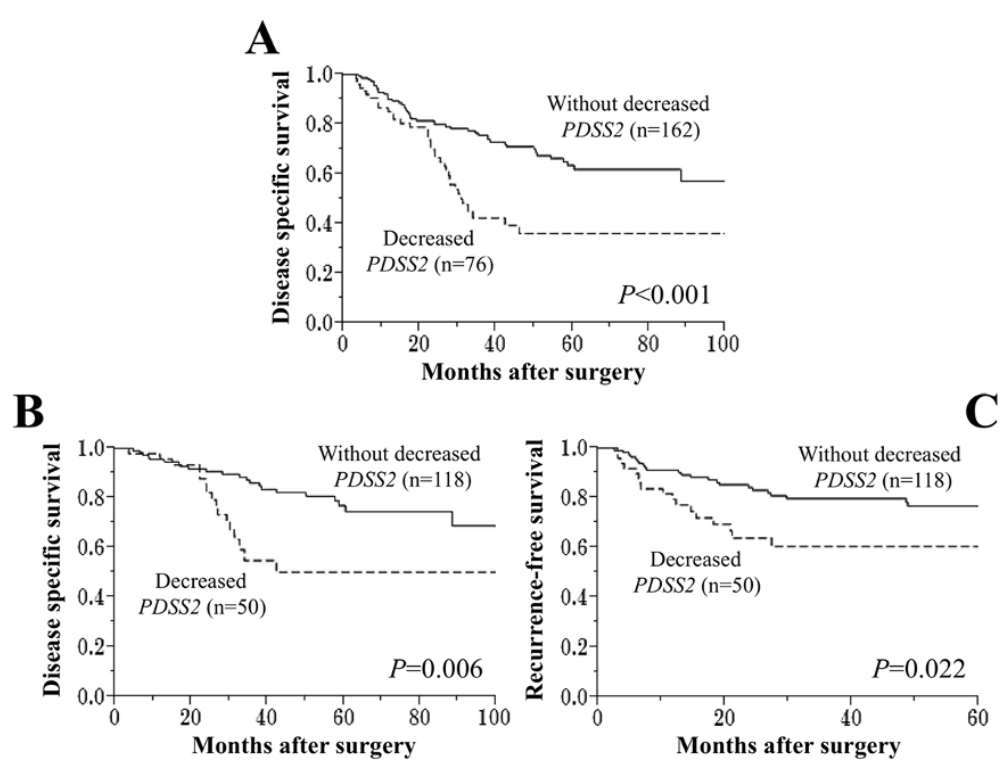

Figure 3 Prognostic implications of PDSS2 mRNA expression in patients with GC. (A) Disease-specific survival of patients with decreased PDSS2 mRNA in GC tissue. (B) (C) Disease-specific (B) and recurrence-free (C) survival among 168 patients who underwent R0 resection.

in the Cox model was assessed with models including time-by-covariate interactions and no significant violations were found in the model.

Of the 168 patients who underwent R0 resection, the disease-specific survival rate was significantly lower for those with decreased PDSS2 mRNA expression in GCs $(\mathrm{n}=50)$ compared with those without $(\mathrm{n}=118)(5$-year survival rates, $50 \%$ and $77 \%$, respectively, $P=0.006$, Figure 3B). Patients with decreased PDSS2 mRNA expression in GCs experienced significantly earlier recurrences after surgery compared with those without (2-year recurrence-free survival rates, $64 \%$ and $84 \%$, respectively, $P=0.022$, Figure $3 C$ ). Initial recurrence sites of 43 relapsing patients with decreased PDSS2 mRNA expression in GCs were peritoneal in 21 (49\%), liver in 6 (14\%), lymph node in 13 (30\%) and others (e.g. lung and bone) in 3 patients. On the other hand, those of 61 relapsing patients without decreased PDSS2 were peritoneal in 35 (57\%), liver in 10 (16\%), lymph node in 8 (13\%) and others in 8 patients. Patients with decreased PDSS2 mRNA expression in GCs were likely to have a node relapse, though it did not reach the statistical significance.

\section{Subgroup analysis of PDSS2 expression according to GC subtype}

Mean PDSS2 mRNA expression levels were equivalent in GC and normal adjacent tissues (Figure 4A). Similarly, the prognostic value of decreased PDSS2 mRNA expression in GCs was comparable among the three GC subtypes (Figure 4B).

\section{Discussion}

PDSSs are heterotetrameric enzymes comprising subunits encoded by PDSS1 (10p12.1) and PDSS2 [10,12]. PDSS activity requires both subunits $[10,14,15]$. The association of PDSS2 with GC was considered because of its chromosomal location (6q21), and because of its inactivation or loss from certain malignancies $[16,26]$. Here we show that PDSS2 mRNA was heterogeneously expressed in GC cell lines, and its expression was inhibited in $73 \%$ and $32 \%$ of GC cell lines and tumor tissues, respectively. We detected hypermethylation of the PDSS2 promoter in five (45\%) of 11 GC cell lines with significantly decreased levels of PDSS2 expression. Further, PDSS2 transcription was reactivated after cells were treated with an inhibitor of DNA methylation. These findings are the first to our knowledge to show that promoter hypermethylation regulates PDSS2 transcription. However, PDSS2 expression was decreased in some GC cells without hypermethylation. Because chromosome $6 \mathrm{q}$ is a frequent site of $\mathrm{LOH}$ in $\mathrm{GC}[17,26,27], \mathrm{LOH}$ may regulate PDSS2 expression as well.

There has been a report demonstrating that PDSS2 was expressed at decreased or undetectable expression in a small number of biopsy GC samples [18]. In the present study, we analyzed 238 surgical specimens of tumors and the corresponding uninvolved tissue to gain further insight into the clinical significance of PDSS2 expression in GC. Consistent with analyses of malignant melanoma and lung cancer [11,12], most patients with GC harbored a decreased level of PDSS2 mRNA in GC tissues, and the mean PDSS2 expression level was significantly decreased in GC compared with normal adjacent tissues. IHC was conducted to determine whether the mRNA level reflected PDSS2 
Table 1 Association between expression level of PDSS2 mRNA and clinicopathological parameters of 238 patients

\begin{tabular}{lccc}
\hline Variables & $\begin{array}{c}\text { Decreased } \text { PDSS2 } \\
\text { mRNA in GC tissues }(\mathbf{n})\end{array}$ & $\begin{array}{c}\text { Others } \\
(\mathbf{n})\end{array}$ & $\begin{array}{c}\boldsymbol{P} \\
\text { value }\end{array}$ \\
\hline Age & & & 0.408
\end{tabular}

$<65$ year

$\geq 65$ year

Gender

Male

Female

Subtype

Proximal nondiffuse

Diffuse

Distal nondiffuse

Carcinoembryonic

antigen ( $\mathrm{ng} / \mathrm{ml})$

$\leq 5$

$>5$

Carbohydrate antigen

19-9 (IU/ml)

$\leq 37$

$>37$

Tumor size (mm)

$<50$

$\geq 50$

Tumor depth (UICC)

pT1

pT2

pT3

pT4

Differentiation

Differentiated

Undifferentiated

Lymphatic involvement

Absent

Present

Vessel invasion

Absent

Present

Infiltrative growth type

Invasive growth

Expansive growth

Lymph node

metastasis (UICC)

\section{pNO}

pN1

pN2

pN3
29

71

91

$$
0.551
$$

120

42

22

14

40
Table 1 Association between expression level of PDSS2 mRNA and clinicopathological parameters of 238 patients (Continued)

\begin{tabular}{lccc}
\hline $\begin{array}{l}\text { Peritoneal lavage } \\
\text { cytology }\end{array}$ & & & 0.306 \\
Negative & 57 & 131 & \\
Positive & 19 & 31 & \\
Distant metastasis (UICC) & & & 0.228 \\
M0 & 50 & 119 & \\
M1 & 26 & 43 & \\
\hline
\end{tabular}

0.298 *Statistically significant $(P<0.05)$. GC, gastric cancer; UICC, Union for International Cancer Control.

protein expression. Because the IHC results indicated that the mRNA data were consistent with the protein level, subsequent analyses were performed according to the mRNA data, which are more amenable to the quantitative analysis $[7,23]$.

Decreased PDSS2 mRNA expression in GCs was sig$0.015^{*}$ nificantly associated with elevated preoperative CA19-9 levels and lymph node metastasis and was identified as an independent prognostic factor. Moreover, patients with decreased PDSS2 mRNA expression in GC tissue experienced significantly earlier recurrence after $\mathrm{R} 0$ resection. Recently, Chen et al. investigated the tumor-suppressing activity of PDSS2 in lung cancer [28]. They reported that the forced overexpression of PDSS2 caused massive cell death through apoptotic pathways and significantly inhibited colony formation and there was an inverse correlation between PDSS2 expression and gelsolin expression, which is known to inhibit apoptosis and enhance cell invasion and metastasis [29], though PDSS2 did not influence the sensitivity of the cancer cells to chemotherapeutic drugs [28]. This tumor suppressive mechanism of PDSS2 might be applied to GC as well.

The expression pattern of PDSS2 mRNA and its prognostic impact were similar among the three GC subtypes (proximal nondiffuse, diffuse, and distal nondiffuse), indicating that PDSS2 expression influences the pathogenesis of all types of GC. Shah et al. reported that one-third of amplified genes, possibly including PDSS2, showed equivalent expression pattern among the three GC subtypes [9].

$0.443 \mathrm{GC}$ is one of the tumors with a high frequency of aberrant methylation, and it frequently exhibits the CpG island methylator phenotype [30,31]. The expression of a large number of genes is suppressed by $\mathrm{CpG}$ island hypermethylation in GC cells, including those encoding tumor suppressors, cell cycle regulators, inducers and executioners of apoptosis, proteins that promote the invasive phenotype, and DNA mismatch repair enzymes [32]. These epigenetic alterations can serve as biomarkers that illuminate an increased metastatic potential 
Table 2 Prognostic factors for disease-specific survival of 238 patients

\begin{tabular}{|c|c|c|c|c|c|c|c|}
\hline \multirow[t]{2}{*}{ Variables } & \multirow[t]{2}{*}{ n (\%) } & \multicolumn{3}{|c|}{ Univariate } & \multicolumn{3}{|c|}{ Multivariable } \\
\hline & & Hazard ratio & $95 \% \mathrm{Cl}$ & $P$ value & Hazard ratio & $95 \% \mathrm{Cl}$ & $P$ value \\
\hline Age $(\geq 65)$ & $138(58 \%)$ & 1.04 & $0.67-1.61$ & 0.843 & & & \\
\hline Gender (female) & $59(25 \%)$ & 1.29 & $0.79-2.05$ & 0.301 & & & \\
\hline Subtype (distal nondiffuse) & $136(57 \%)$ & 0.43 & $0.28-0.67$ & $<0.001$ & 0.64 & $0.40-1.01$ & 0.056 \\
\hline Carcinoembryonic antigen (>5 ng/ml) & $47(20 \%)$ & 1.48 & $0.86-2.42$ & 0.149 & & & \\
\hline Carbohydrate antigen 19-9 (>37 IU/ml) & $44(18 \%)$ & 1.98 & $1.17-3.20$ & 0.012 & 1.23 & $0.71-2.06$ & 0.445 \\
\hline Tumor size ( $\geq 50 \mathrm{~mm})$ & $129(54 \%)$ & 2.86 & $1.78-4.80$ & $<0.001$ & 1.40 & $0.83-2.42$ & 0.211 \\
\hline Tumor depth (pT4, UICC) & $110(46 \%)$ & 4.17 & $2.61-6.88$ & $<0.001$ & 1.38 & $0.78-2.50$ & 0.273 \\
\hline Tumor differentiation (undifferentiated) & 139 (58\%) & 2.03 & $1.28-3.32$ & 0.002 & 1.45 & $0.83-2.60$ & 0.197 \\
\hline Lymphatic involvement & $203(85 \%)$ & 6.31 & $2.36-25.8$ & $<0.001$ & 1.45 & $0.45-6.50$ & 0.559 \\
\hline Vessel invasion & $134(56 \%)$ & 2.65 & $1.66-4.37$ & $<0.001$ & 1.75 & $1.07-2.97$ & $0.026^{*}$ \\
\hline Invasive growth & $83(35 \%)$ & 3.03 & $1.97-4.70$ & $<0.001$ & 1.19 & $0.70-2.05$ & 0.520 \\
\hline Lymph node metastasis & $149(63 \%)$ & 7.02 & $3.70-15.1$ & $<0.001$ & 1.38 & $0.56-3.81$ & 0.503 \\
\hline Peritoneal lavage cytology (positive) & $50(21 \%)$ & 4.33 & $2.76-6.74$ & $<0.001$ & 1.87 & $1.14-3.06$ & $0.014^{*}$ \\
\hline UICC stage (III-IV) & $140(59 \%)$ & 9.68 & $4.97-21.8$ & $<0.001$ & 2.63 & $0.94-7.83$ & 0.065 \\
\hline Decreased PDSS2 mRNA in GCs & $76(32 \%)$ & 2.18 & $1.40-3.37$ & $<0.001$ & 1.91 & $1.19-3.04$ & $0.008^{*}$ \\
\hline
\end{tabular}

*Statistically significant in multivariate analysis. GC, gastric cancer; $\mathrm{Cl}$, confidence interval; UICC, Union for International Cancer Control.

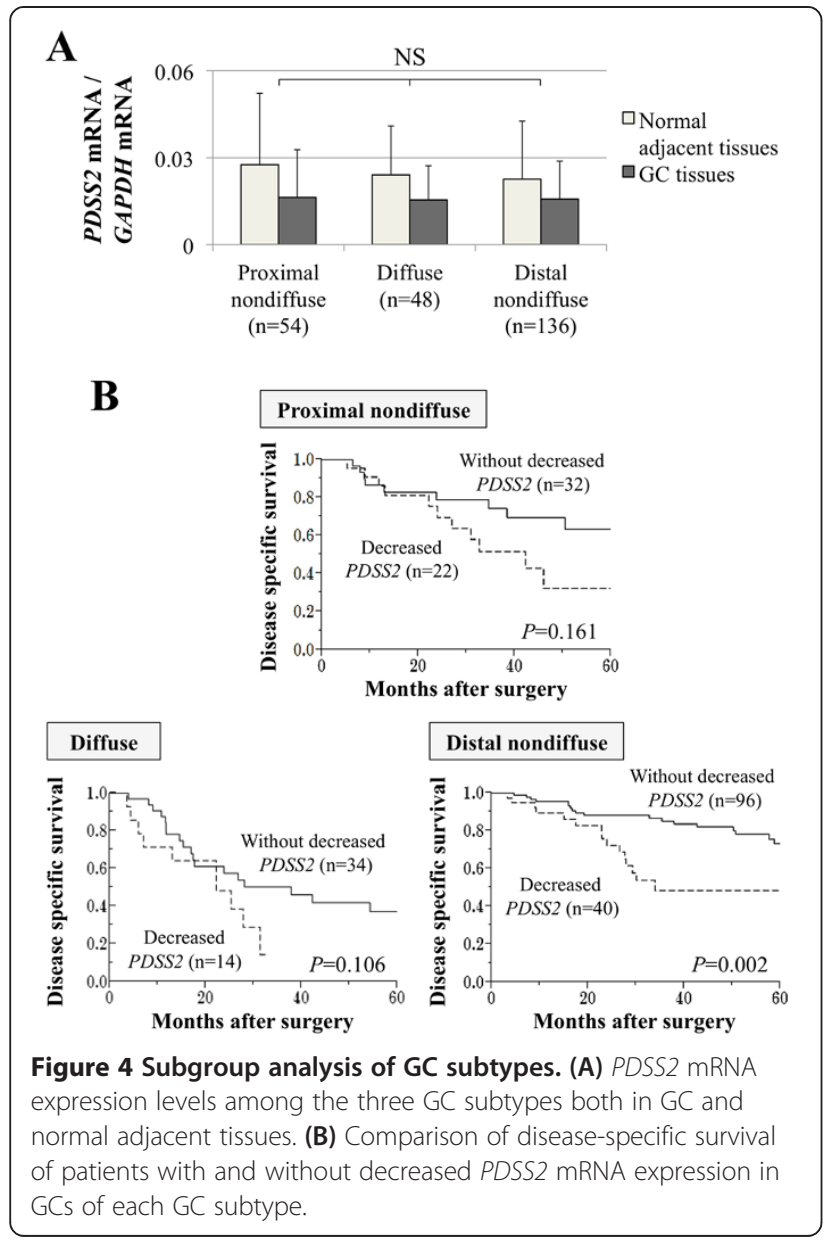

and aggressive tumor phenotype [33] as well as therapeutic targets [34]. Therefore, identification of other genes that are regulated by methylation in GC cells will likely improve the management of GC.

The tumor suppressive function of PDSS2 are supported by the present findings as follows: (1) decreased expression of PDSS2 was frequently detected in GC tissues, (2) the mean level of PDSS2 expression was significantly lower in GC tissues, and (3) decreased expression of PDSS2 was associated with early recurrence and subsequent poor prognosis. PDSS2 expression levels in biopsy tissue obtained using endoscopic surveillance samples or in surgical specimens may be useful for predicting early recurrence and poor prognosis, which will likely aid efforts to design more efficacious therapeutic strategies.

This study was limited by its lack of sufficient functional analysis of PDSS2, which tempers the conclusion that it acts as a tumor suppressor in GC. Further studies including pathway analysis in gastric carcinogenesis and functional analysis are expected to clarify the molecular mechanisms underlying the biological activities of PDSS2 in GC.

\section{Conclusion}

In conclusion, our findings support the conclusion that the expression of the putative tumor suppressor gene PDSS2 is regulated by promoter hypermethylation in GC cells and indicate. Our results indicate further that decreased expression of PDSS2 mRNA may represent a novel biomarker for progression and recurrence of all types of GC. 


\section{Additional file}

Additional file 1: Table S1. Primers and annealing temperature.

\section{Competing interests}

The authors declare that they have no competing interests.

\section{Authors' contributions}

MK, HO, FS, DS, HT, RH and KM performed experiments and data analysis. DK, CT, SY, TF, GN, HS, MK, MF and YK collected cases and clinical data. MK and $\mathrm{SN}$ conceived and designed the study, and prepared the initial manuscript. YK supervised the project. All authors contributed to the final manuscript. All authors read and approved the final manuscript.

\section{Acknowledgements}

The authors thank Naoki Iwata for his support to collect clinical data.

\section{Author details}

${ }^{1}$ Department of Gastroenterological Surgery (Surgery II), Nagoya University Graduate School of Medicine, 65 Tsurumai-cho, Showa-ku, Nagoya 466-8550, Japan. ${ }^{2}$ Center for Advanced Medicine and Clinical Research, Nagoya University Hospital, Nagoya, Japan.

Received: 27 July 2014 Accepted: 3 October 2014

Published online: 22 October 2014

\section{References}

1. Wang Z, Wang J, Yang Y, Hao B, Wang R, Li Y, Wu Q: Loss of has-miR-337$3 p$ expression is associated with lymph node metastasis of human gastric cancer. J Exp Clin Cancer Res 2013, 32:76.

2. Jemal A, Bray F, Center MM, Ferlay J, Ward E, Forman D: Global cancer statistics. CA Cancer J Clin 2011, 61:69-90.

3. Leung WK, Wu MS, Kakugawa Y, Kim JJ, Yeoh KG, Goh KL, Wu KC, Wu DC, Sollano J, Kachintorn U, Gotoda T, Lin JT, You WC, Ng EK, Sung JJ: Screening for gastric cancer in Asia: current evidence and practice. Lancet Oncol 2008, 9:279-287.

4. Kanda M, Nomoto S, Oya H, Shimizu D, Takami H, Hibino S, Hashimoto R, Kobayashi D, Tanaka C, Yamada S, Fujii T, Nakayama G, Sugimoto H, Koike M, Fujiwara M, Kodera Y: Dihydropyrimidinase-like 3 facilitates malignant behavior of gastric cancer. J Exp Clin Cancer Res 2014, 33:66.

5. Wang YY, Li L, Zhao ZS, Wang YX, Ye ZY, Tao HQ: L1 and epithelial cell adhesion molecules associated with gastric cancer progression and prognosis in examination of specimens from 601 patients. J Exp Clin Cancer Res 2013, 32:66.

6. Resende C, Thiel A, Machado JC, Ristimaki A: Gastric cancer: basic aspects Helicobacter 2011, 16(Suppl 1):38-44.

7. Kanda M, Shimizu D, Nomoto S, Takami H, Hibino S, Oya H, Hashimoto R, Suenaga M, Inokawa Y, Kobayashi D, Tanaka C, Yamada S, Fujii T, Nakayama G, Sugimoto $H$, Koike M, Fujiwara M, Kodera Y: Prognostic impact of expression and methylation status of DENN/MADD domain-containing protein 2D in gastric cancer. Gastric Cancer 2014, Epub ahead of print, PubMed PMID: 24695972.

8. Lauren P: The two histological main types of gastric carcinoma: diffuse and so-called intestinal-type carcinoma. An attempt at a histo-clinical classification. Acta Pathol Microbiol Scand 1965, 64:31-49.

9. Shah MA, Khanin R, Tang L, Janjigian YY, Klimstra DS, Gerdes H, Kelsen DP: Molecular classification of gastric cancer: a new paradigm. Clin Cancer Res 2011, 17:2693-2701

10. Saiki R, Nagata A, Kainou T, Matsuda H, Kawamukai M: Characterization of solanesyl and decaprenyl diphosphate synthases in mice and humans. Febs j 2005, 272:5606-5622.

11. Chen P, Yu J, Knecht J, Chen Q: Decrease of PDSS2 expression, a novel tumor suppressor, in non-small cell lung cancer. Cancer Epidemio/ 2013, 37:166-171.

12. Fung JM, Smith R, Brown MA, Lau SH, Xie D, Lau GK, Guan XY: Identification and characterization of a novel melanoma tumor suppressor gene on human chromosome 6q21. Clin Cancer Res 2009, 15:797-803.

13. Quinzii CM, DiMauro S, Hirano M: Human coenzyme Q10 deficiency. Neurochem Res 2007, 32:723-727.
14. DiMauro S, Quinzii CM, Hirano M: Mutations in coenzyme Q10 biosynthetic genes. J Clin Invest 2007, 117:587-589.

15. Turunen M, Olsson J, Dallner G: Metabolism and function of coenzyme Q. Biochim Biophys Acta 2004, 1660:171-199.

16. Li BC, Chan WY, Li CY, Chow C, Ng EK, Chung SC: Allelic loss of chromosome $6 q$ in gastric carcinoma. Diagn Mol Pathol 2003, 12:193-200.

17. Carvalho B, Seruca R, Carneiro F, Buys CH, Kok K: Substantial reduction of the gastric carcinoma critical region at $6 q 16.3-q 23.1$. Genes Chromosomes Cancer 1999, 26:29-34.

18. Chen P, Zhao SH, Chu YL, Xu K, Zhu L, Wu Y, Song J, Cao CX, Xue X, Niu YY: Anticancer activity of PDSS2, prenyl diphosphate synthase, subunit 2, in gastric cancer tissue and the SGC7901 cell line. Anticancer Drugs 2009, 20:141-148.

19. Sobin LH, Gospodarowicz MK, C W: International Union Against Cancer, TNM Classification of Malignant Tumors. 7th edition. New York: Wiley-Blackwell; 2009.

20. Sakuramoto S, Sasako M, Yamaguchi T, Kinoshita T, Fujii M, Nashimoto A, Furukawa $\mathrm{H}$, Nakajima $\mathrm{T}$, Ohashi $\mathrm{Y}$, Imamura $\mathrm{H}$, Higashino M, Yamamura $\mathrm{Y}$, Kurita A, Arai K: Adjuvant chemotherapy for gastric cancer with S-1, an oral fluoropyrimidine. N Engl J Med 2007, 357:1810-1820.

21. Kanda M, Nomoto S, Oya H, Takami H, Hibino S, Hishida M, Suenaga M, Yamada S, Inokawa Y, Nishikawa Y, Asai M, Fujii T, Sugimoto H, Kodera Y: Downregulation of DENND2D by promoter hypermethylation is associated with early recurrence of hepatocellular carcinoma. Int J Oncol 2014, 44:44-52.

22. Kanda M, Nomoto S, Okamura Y, Nishikawa Y, Sugimoto $H$, Kanazumi N, Takeda S, Nakao A: Detection of metallothionein $1 \mathrm{G}$ as a methylated tumor suppressor gene in human hepatocellular carcinoma using a novel method of double combination array analysis. Int J Oncol 2009, 35:477-483

23. Kanda M, Shimizu D, Nomoto S, Hibino S, Oya H, Takami H, Kobayashi D, Yamada S, Inokawa Y, Tanaka C, Fujii T, Sugimoto H, Koike M, Fujiwara M, Kodera $Y$ : Clinical significance of expression and epigenetic profiling of TUSC1 in gastric cancer. J Surg Oncol 2014, 110:136-144.

24. Takai D, Jones PA: The CpG island searcher: a new WWW resource. In Silico Biol 2003, 3:235-240.

25. Kanda M, Nomoto S, Okamura Y, Hayashi M, Hishida M, Fujii T, Nishikawa Y Sugimoto H, Takeda S, Nakao A: Promoter hypermethylation of fibulin 1 gene is associated with tumor progression in hepatocellular carcinoma. Mol Carcinog 2011, 50:571-579.

26. Queimado L, Seruca R, Costa-Pereira A, Castedo S: Identification of two distinct regions of deletion at $6 \mathrm{q}$ in gastric carcinoma. Genes Chromosomes Cancer 1995, 14:28-34.

27. Nagai H, Pineau P, Tiollais P, Buendia MA, Dejean A: Comprehensive allelotyping of human hepatocellular carcinoma. Oncogene 1997, 14:2927-2933.

28. Chen P, Zhang Y, Polireddy K, Chen Q: The tumor-suppressing activity of the prenyl diphosphate synthase subunit 2 gene in lung cancer cells. Anticancer Drugs 2014, 25:790-798.

29. An JH, Kim JW, Jang SM, Kim CH, Kang EJ, Choi KH: Gelsolin negatively regulates the activity of tumor suppressor p53 through their physical interaction in hepatocarcinoma HepG2 cells. Biochem Biophys Res Commun 2011, 412:44-49.

30. Jang BG, Kim WH: Molecular pathology of gastric carcinoma. Pathobiology 2011, 78:302-310.

31. Kim TY, Jong HS, Jung Y, Kim TY, Kang GH, Bang YJ: DNA hypermethylation in gastric cancer. Aliment Pharmacol Ther 2004, 20(Suppl 1):131-142.

32. To KF, Leung WK, Lee TL, Yu J, Tong JH, Chan MW, Ng EK, Chung SC, Sung JJ: Promoter hypermethylation of tumor-related genes in gastric intestinal metaplasia of patients with and without gastric cancer. Int J Cancer 2002, 102:623-628.

33. Janjigian YY, Kelsen DP: Genomic dysregulation in gastric tumors. J Surg Oncol 2013, 107:237-242

34. Bird A: Perceptions of epigenetics. Nature 2007, 447:396-398

\section{doi:10.1186/s13046-014-0088-3}

Cite this article as: Kanda et al:: Decreased expression of prenyl diphosphate synthase subunit 2 correlates with reduced survival of patients with gastric cancer. Journal of Experimental \& Clinical Cancer Research 2014 33:88. 